\title{
Base de données Égalité homme- femme, Institutions et Développement
}

par Johannes Jütting, Christian Morrisson, Jeff Dayton-Johnson et Denis Drechsler

- Les récentes expériences de la Finlande, de l'Allemagne, du Chili et du Libéria montrent que les femmes peuvent occuper des postes clés au sein des gouvernements.

- Cependant, certaines traditions et normes socio-économiques enferment certains pays dans une trappe de pauvreté en excluant les femmes de certaines activités économiques - davantage de discrimination, moins de croissance, davantage de pauvreté !

- Les stratégies menées avec cohérence, en respectant la culture et en incluant les femmes, font disparaitre les pratiques discriminatoires qui sont contre-productives.

L'EID - une ressource de qualité pour améliorer les politiques

La base de données Égalité homme-femme, Institutions et Développement - EID (www.oecd.org/dev/institutions/ basededonneesEID), représente un nouvel instrument pour les chercheurs et responsables politiques qui leur permet de connaître et d'analyser les obstacles à la participation des femmes au développement économique.

Cette base comporte un ensemble de 162 pays et comprend 50 indicateurs pour mesurer la discrimination envers les femmes. La base a été construite à partir de sources diverses et elle combine d'une manière systématique et cohérente les données empiriques courantes qui existent sur le statut socio-économique des femmes. Son caractère innovateur tient à la prise en compte des variables institutionnelles qui vont des comportements à l'intérieur des ménages aux normes sociales. Les informations sur les pratiques culturelles et traditions qui ont une incidence sur la participation des femmes au développement sont codées afin de mesurer le niveau de discrimination. Une telle synthèse des variables liées aux relations homme femme et
I'accent particulier mis dans cette base sur les institutions sociales rendent cette base unique, en fournissant une boite à outils pour un grand nombre de recherches analytiques et en permettant une adaptation au cas par cas aux questions particulières ou aux problèmes politiques:

Qu'est-ce qui distingue I'EID des autres bases de données existantes?

La discrimination envers les femmes comporte de multiples facettes. Il est de plus en plus évident que les institutions sociales qui comportent des lois non-écrites, des coutumes et des traditions, sont des facteurs importants de détermination du degré de participation des femmes à la vie sociale et économique. Par conséquent, l'EID créé un ensemble d'indicateurs innovants qui peuvent être regoupés en quatre catégories :

i) Code familial : comprend des informations relatives aux coutumes maritales (âge, héritage et polygamie) et au pouvoir de décision au sein du foyer (autorité parentale, répudiation). 
ii) Intégrité physique : pointe les cas de violence envers les femmes par le biais de pratiques traditionnelles comme la mutilation génitale des femmes ou d'autres atteintes physiques (par exemple, viol, agressions, harcèlement).

iii) Libertés civiques : mesurent la capacité des femmes à prendre part à la vie sociale, soit en étant candidates à des fonctions politiques, soit en étant autorisées à sortir librement de chez elles (par exemple, sans porter de voile ni être escortées par un homme de leur famille).

iv) Droits de propriété : indiquent la qualité des droits économiques de base des femmes - être propriétaire, avoir accès aux prêts bancaires, à la terre ou à d'autres biens matériels.

\section{Application et emploi de l'EID}

Les analyses préliminaires qui utilisent cette base montrent clairement la pertinence des institutions sociales pour comprendre le rôle économique des femmes. Elles indiquent très clairement que plus les institutions sociales sont discriminatoires, plus les taux de participation des femmes au marché du travail sont faibles (Figure 1).

\section{Figure 1. Institutions sociales et la participation} des femmes au marché du travail

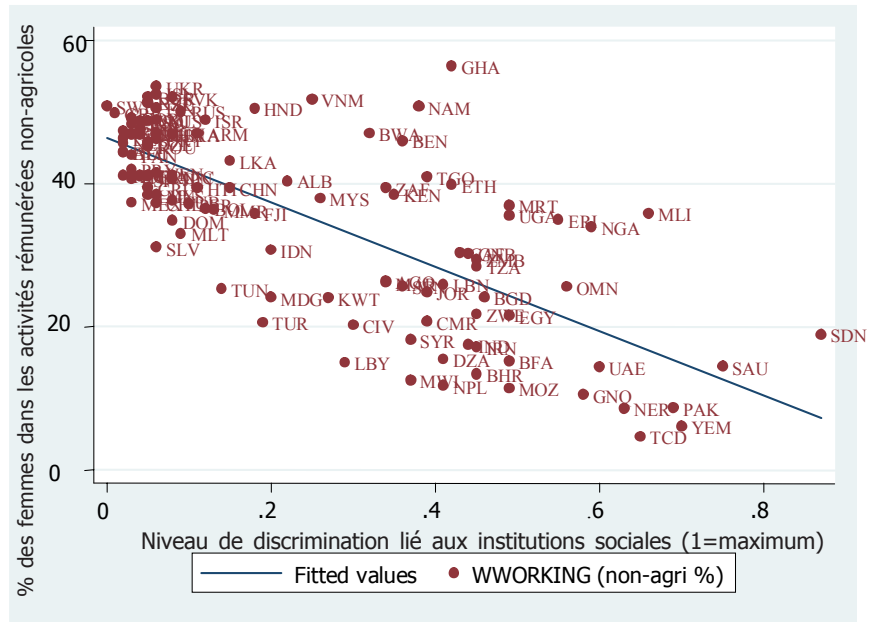

La variable explicative utilise la moyenne arithmétique de tous les sous-secteurs des institutions sociales; la valeur $1(0)$ indique le plus haut (bas) niveau de discrimination.

Source : Jütting et al. (2006).
Les institutions sociales discriminatoires ont un impact direct sur la participation des femmes au développement économique. Par exemple, un faible niveau des droits de propriété bloque le développement des entreprises dirigées par les femmes. Ces institutions exercent aussi une influence indirecte par leurs effets sur l'accès des femmes aux ressources; les violences à l'égard des femmes compromettent leur santé et ainsi leur chance d'accès au marché du travail. Ces effets ont des conséquences négatives sur la participation des femmes dans l'économie.

\section{Dans le futur}

La base de données Égalité homme-femme, Institutions et Développement (EID) contribue de manière importante au débat sur le genre, notamment parce qu'elle inclut les obstacles au développement économique des femmes. Notre analyse économétrique initiale suggère que le poids des facteurs institutionnels l'emporte sur des variables plus fréquemment utilisées telles que le revenu par tête. Cette découverte va dans le sens de l'idée selon laquelle une amélioration du rôle économique des femmes ne suivra pas nécessairement une augmentation du niveau de revenu. Au lieu de cela, certaines mesures politiques - comme les récentes réformes du Code de la famille au Maroc - doivent être mises en place pour remédier directement aux goulets d'étranglement institutionnels. De ce point de vue-là, I'EID permet d'identifier les politiques qui s'attaquent aux causes plutôt qu'aux symptômes de la discrimination liée au genre.

À lire :

Jütting, J., C. Morrisson, J. Dayton-Johnson et D. Drechsler, Measuring Gender (In)Equality, Working Paper No. 247, Centre de développement de l'OCDE.

www.oecd.org/dev/reperes www.oecd.org/dev/cahiers www.oecd.org/dev/dt 